\title{
Pedunculated solitary fibrous tumours arising from the pleura
}

\author{
A. Poyraz1, D. Kilic1, A. Hatipoglu1, T. Bakirci2, B. Bilezikci3
}

ABSTRACT: Pedunculated solitary fibrous tumours arising from the pleura. A. Poyraz, D. Kilic, A. Hatipoglu, T. Bakirci, B. Bilezikci.

Solitary fibrous tumour (SFT) is one of the rare tumours which arise from visceral pleura. Klemperer and Rabin first described SFT as a distinct clinical entity among primary pleural tumoUrs in 1931.

Approximately 820 cases have been reported in literature to date. The management of patients with SFT is complete resection of the tumour and follow up of the patient to detect any possible late recurrence. In the present paper, we report two cases of pedunculated solitary fibrous tumours of the pleura that appeared as a wandering chest nodule to which surgical resection undertaken at our hospital.

The aim is to summarise our experience in the management of solitary fibrous tumour.

Monaldi Arch Chest Dis 2006; 65: 3, 165-168.

Keywords: Pleura, neoplasm, thorax.

1 Department of Thoracic and Cardiovascular Surgery.

2 Department of Chest Medicine and Tuberculosis.

3 Department of Pathology. Faculty of Medicine, Baskent University, Ankara, Turkey.

Correspondence: A. Serhan Poyraz, Department of Thoracic and Cardiovascular Surgery, Faculty of Medicine, Baskent University, Konya,Turkey; e-mail: ahmetserhanpoyraz@hotmail.com

\section{Introduction}

Pedunculated solitary fibrous tumours (SFT) are slow-growing, localised and somewhat rare tumours of pleura. However, they may occasionally behave aggressively. They are derived from submesothelial mesenchymal cells. It occurs in both sexes and in a wide range of ages, but has a peak incidence in individuals aged over 50. In 1931, Klemperer and Rabin divided primary tumours of the pleura into two categories: diffuse mesothelioma and localised mesothelioma [1]. Diagnosis of SFT is often suggested initially by its typical radiological findings. SFT shows a solitary, well-circumscribed, pedunculated and pleural-based mass. Blood is supplied to the tumour through the pedicle and arises from feeding bronchial, intercostal or diaphragmatic vessels. Approximately, half the patients with pleural fibromas are asymptomatic but cough, chest pain and dyspnea may present symptoms, particularly if the tumour is large. We report two cases treated in our hospital over a period of 24 months. We discuss the clinical presentation, surgical treatment, and follow up, which includes the differential diagnosis of a moving intrathoracic mass.

\section{Patient 1}

The patient was a 52-year-old woman who was admitted to the emergency unit with complaints of shortness of breath for 15 days. A diagnosis of mass was suspected, based on clinical, radiological and needle biopsy findings. She was hospitalised at the Unit of Thoracic Surgery of our institution for surgery. There was no history of haemoptysis or weight loss but she complained of intermittent chest pains on the left side anteriorly with positional mobility of mass. The onset of the pain was unpredictable, but it was associated with anterior motion of the body. A chest radiograph showed a huge lobulated mass in the left lower zone, which is broadly based on left diaphragm (figure 1). The

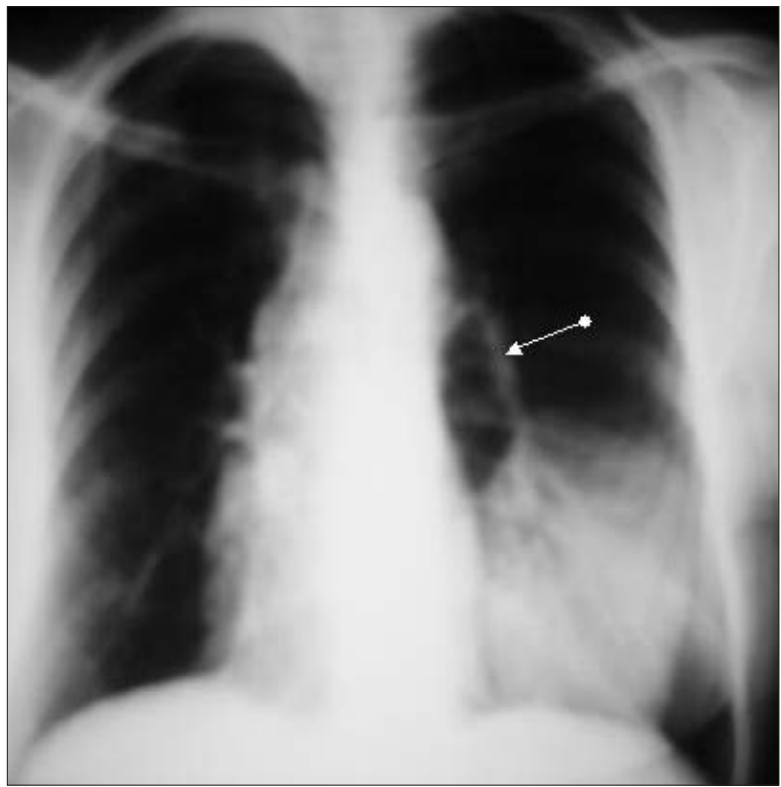

Fig. 1. - Chest radiograph showing huge lobulated mass in the lower left zone. 
lower left cardiac border was straight, suggesting lower left lobe collapse. The pedunculated tumour was changed in appearance with inspiration-expiration or with changes in patient positioning; such mobility is virtually pathognomonic. A Computed Tomography (CT) scan of the thorax showed a huge lobulated mass covering the lower left hemithorax (figure 2). She was a non-smoker and in good health. Laboratory investigations were normal. Results for tumour markers were negative. There was no evidence of any invasion into the neighbouring structures and no calcification was seen within the lesions. The lesion was diagnosed as fibrous tumour of the pleura by histological and immunohistochemical analysis of the material obtained by transthoracic cutting-needle biopsy (figure 3 ). An attempt at fine needle aspiration revealed fragments of fibrous and vascular tissue, with no signs of malignancy. Postero-lateral thoracotomy was performed. The totally excised mass was unilobular and $10 \times 15 \times 5 \mathrm{~cm}$ in diameter and the tumour wall was thin and smooth. A giant, ovoid tumour, which was whitish-gray, stiff, and pedunculated, was found arising from the left inferior lingual segment and projecting into the pleural space. Intra-operative pathologic evaluation did not show malignant or invasive findings. A thick, vascular capsule surrounded the tumour. The tumour and its pedicle were completely resected. Thoracotomy with tumour extirpation exhibited a solitary fibrous tumour of the pleura. She was discharged from hospital on the post-operative fifth day. Post-operative course was uneventful. The patient has completed 24 months of follow-up, without any delayed complication and remains asymptomatic.

\section{Patient 2}

A 69-year old male smoker applied with a 7 day history of cough with dirty white sputum. The patient had a history of thoracic trauma and rib fracture due to traffic accident that the patient was involved in one week ago. The patient's chest radiograph showed a huge lobulated mass in the lower right lobe, which was broadly based on right diaphragm. The lower right cardiac border was straight, suggesting right lower lobe collapse. A CT scan of the thorax showed a huge lobulated mass covering the lower two thirds of the right hemithorax (figure 4). Laboratory investigations were normal. Fiberoptic bronchoscopy showed extrinsic compression of the right bronchial tree, but no endobronchial lesion and cytology was not diagnostic. The totally excised mass, which was on the diaphragm without invasion, was pedunculated and arose from the bottom surface of visceral pleura of the lower right lobe. It was bilobular and $20 \times 15 \times 10 \mathrm{~cm}$ in diameter and the tumour wall was thin and smooth. Macroscopically, it appeared as bulbous, coarse nodules elevated above the surface of the pleura, and pedunculated on pleural-based pedicles that contain hypertrophic arteries and veins. The histopathological examination of the tumour was consistent with a fibrous tumour of the pleura with no evidence of malignancy (figure 5).

\section{Discussion}

Clagett et al first used the term "localised fibrous mesothelioma" in 1952 to distinguish these tumours from the more familiar asbestos-related, diffuse malignant mesothelioma [1]. The tumour most often arises from the visceral pleura in the chest. Cardillo et al found that $87.28 \%$ of solitary fibrous tumours in their series originated in the visceral pleura and only $12.72 \%$ in the parietal pleura [2]. The origin of Solitary fibrous tumour is unclear. No association exists with smoking or asbestos exposure. While some studies indicate that

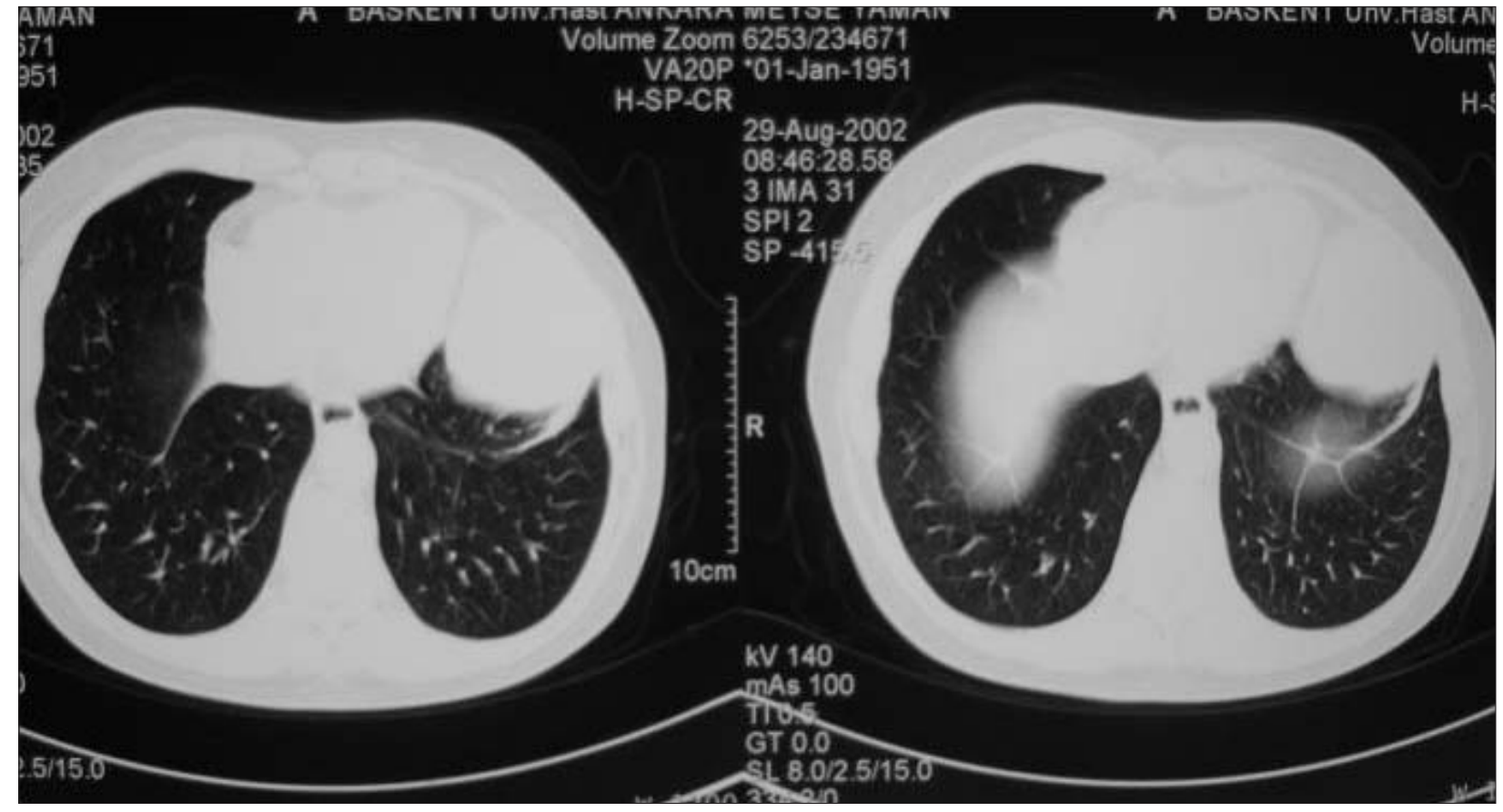

Fig. 2. - A CT scan of the thorax showing a huge lobulated mass filling the lower left lobe 
SFTP is mesothelial in origin, other reports suggest an origin from primitive submesothelial mesenchymal cells distinct from diffuse malignant mesothelioma. The tumour is attached by a short pedicle in our cases and gross pathologic examination revealed a lobulated well-encapsulated mass. The capsule of the mass was greyish white and glistening, it also contained small blood vessels. The severed surface of the mass was yellowish white, septated, and solid. Neither a haemorrhage nor necrosis was present. Microscopically, the tumour had a pattern of a hemangiopericytomatous area alternating with a hypocellular fibrous region; this feature suggested a SFTP. With immunohistochemical staining, the neoplastic cells had a positive reaction to $\mathrm{CD} 34$ and negative reactions to CD31, desmin, cytokeratin, and S-100 protein.

The tumours were classified histologically as benign or malignant according to the following criteria used by England [3]. (1) mitotic count more than 4 mitosis/10 high-power fields (HPF) (3400); (2) presence of necrosis; (3) hypercellularity as judged by nuclear crowding and overlapping; and (4) presence of nuclear atypia. According to this criteria, of the 223 cases in England et al.'s study 141 tumours were benign and 82 tumours were malignant. For the purpose of identifying solitary fibrous tumours of pleura that will behave in a malignant manner, we also applied the more recent criteria of specific immunohistochemical stains, microvessel density and proliferation index. Symptoms, if any, tend to be more common in larger lesions and include either local symptoms (such as chest pain, cough, dyspnea, and more rarely hemoptisis) or systemic symptoms (such as weakness, nocturnal sweating, chills, weight loss, digital clubbing, hypertrophic ostearthropathy, and hypoglicemia). However these patients did not have any para-neoplastic syndromes. When symptoms are present, they are more often multiple, regressing completely after surgical resection, as ob- served in our patients. Most tumours pleuralbased, having sharply circumscribed borders and a round contour, and are less than $10 \mathrm{~cm}$ in diameter. A few are located in the fissures. Calcification is rare. On MRI the lesions signal characteristics reflect its hypocellularity and abundant collagen stroma. The lesion typically has low signal intensity on T1 weighted images. Heterogeneous, but predominantly low signal is also seen on proton weighted images and T2 weighted images [4]. Areas of high signal intensity correspond to regions of necrosis or myxoid degeneration within the lesion. Intense lesion enhancement can be seen following the administration of gadolinium. The differential diagnosis is wide-ranging and complex in many cases, can have similar clinical and radiographic signs. Transthoracic CT-guided biopsy does not seem to be a reliable diagnostic method [5]. But making the diagnosis of solitary fibrous tumour of the pleura by cutting-needle biopsy permits a proper and cost-effective allocation of medical resources. Articles have been published pro-

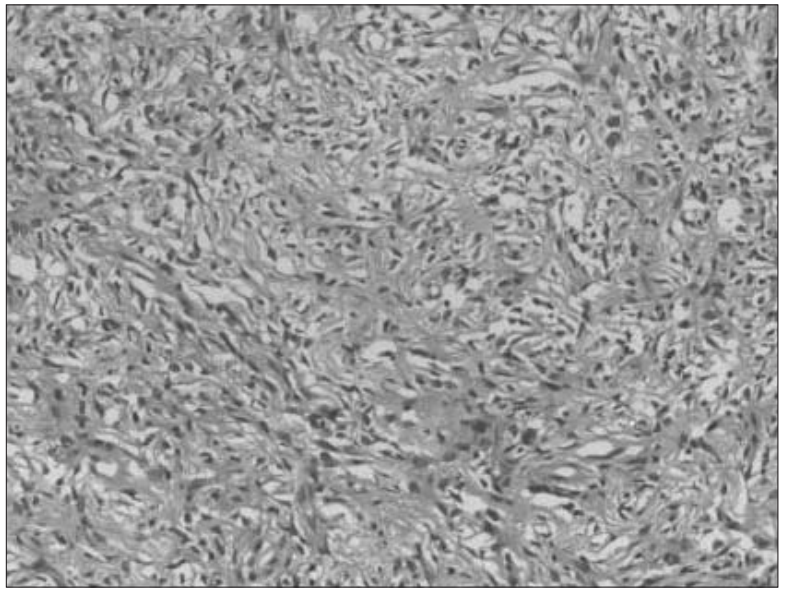

Fig. 3. - (H\&E, x200) Spindle cell proliferation. There is no atypia or necrosis. Mitotic count is $2 / 10 \mathrm{HPF}$

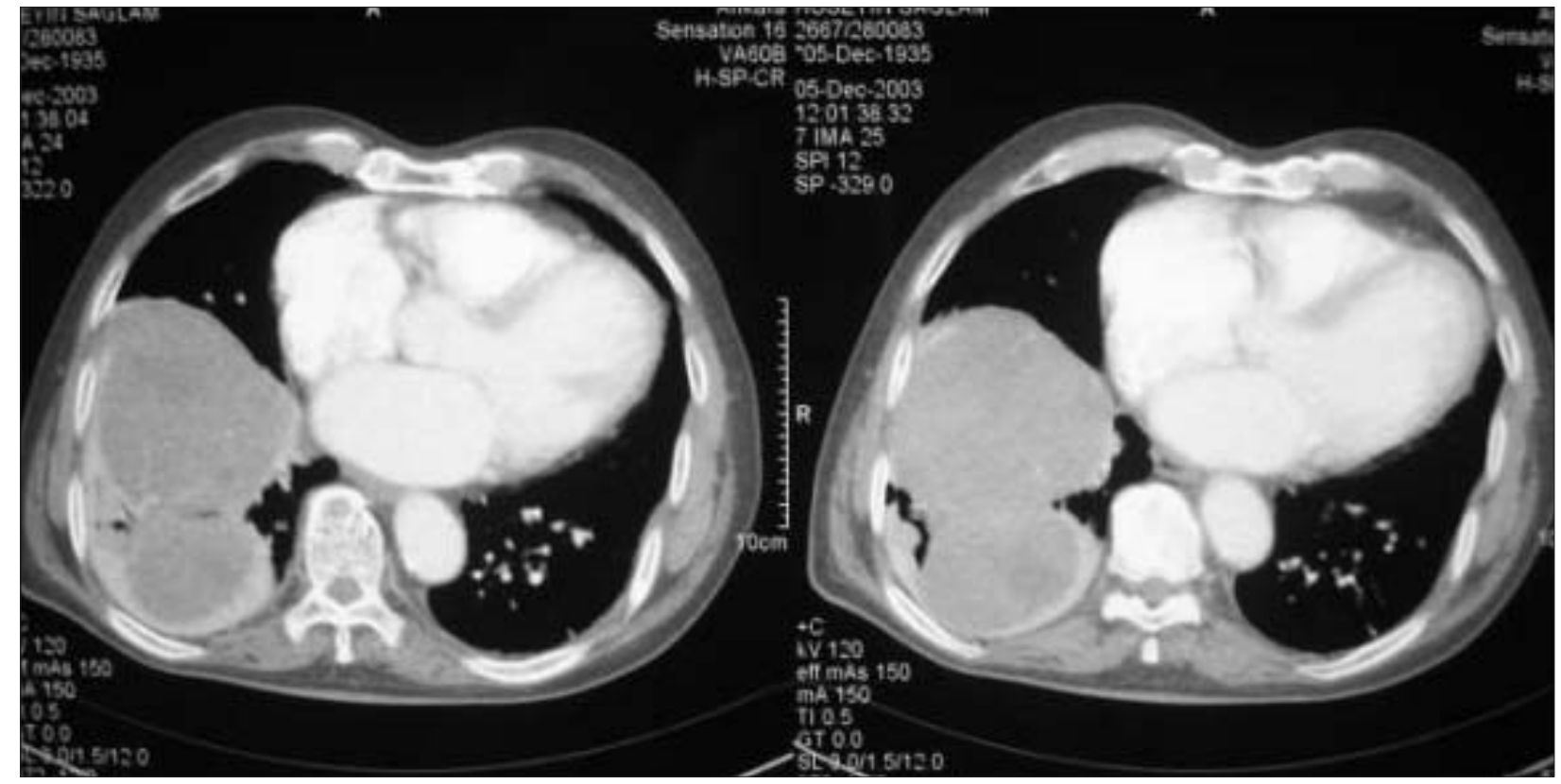

Fig. 4. - A CT scan of the thorax showing a huge lobulated mass filling the lower right lobe. 


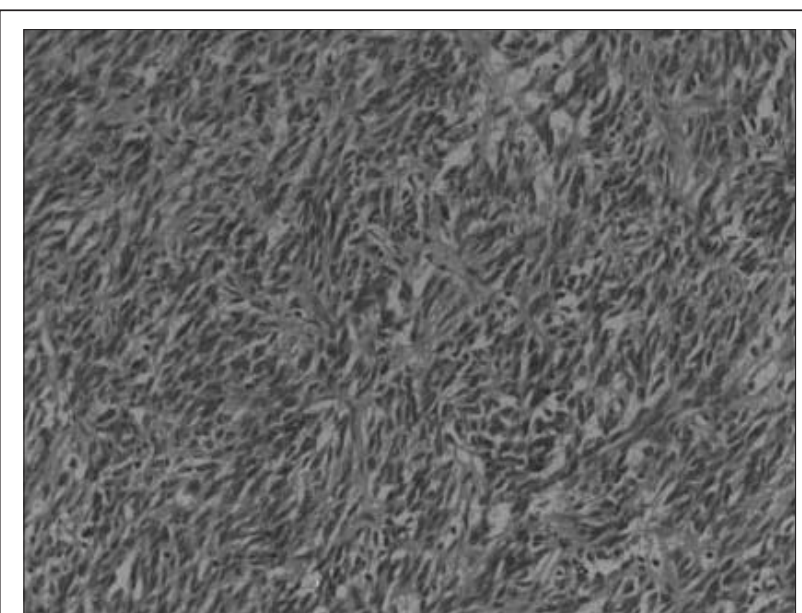

Fig. 5. - (H\&E, x200) Spindle cell proliferation. There is no atypia, mitotic activity or necrosis

viding strong evidence that a confident preoperative diagnosis of fibrous tumour of the pleura can be made by histologic and immunohistochemical analysis of material obtained by transthoracic cutting-needle biopsy $[6,7]$. Congregado et al. believe that all undiagnosed thoracic tumours should first be evaluated by video-assisted thoracoscopy [8]. However, we agree with the opinion of Tastepe et al. [9], because radiologic imaging cannot differentiate benign from malignant lesions with $100 \%$ accuracy, surgical biopsy remains the gold standard for diagnosis. On thoracotomy, surgical therapy proved curative and emphasises early intervention in all cases. Local recurrences and malignant degeneration of previously benign tumour can occur years after seemingly adequate surgical treatment $[10,11]$.

Based on our experience, it was concluded that making the definitive diagnosis allows for a proper planning of a curative thoracotomy and the treatment of choice for these tumours at present is surgical resection, and long-term follow up is needed, even in cases of tumour classified as histologically benign, because of the possible risk of recurrence and malignant transformation.

\section{References}

1. Rena O, Filosso PL, Papalia E, et al. Solitary fibrous tumour of the pleura: surgical treatment. Eur J Cardiothor Surg 2001; 19:185-189.

2. Cardillo G, Facciolo F, Cavazzana AO, Capece G, Gasparri R, Martelli M. Localized (solitary) fibrous tumours of the pleura: an analysis of 55 patients. Ann Thorac Surg 2000; 70: 1808-12.

3. England DM, Hocchholzer L, Mc Carthy MJ. Localized benign and malignant fibrous tumor of the pleura. A clinicopathologic review of 223 cases. Am J Surg Pathol 1989; 13: 640-58.

4. Truong M, Munden RF, Kemp BL. Localized fibrous tumor of the pleura. AJR 2000; 174: 42.

5. Altinok T, Topçu S, Tastepe AI, Yazici U, Cetin G. Localized fibrous tumors of the pleura: clinical and surgical evaluation. Ann Thorac Surg 2003; 76: 892-5.

6. Collard P, Weynand B. Confident diagnosis of solitary fibrous tumor of the pleura using cutting-needle biopsy. Chest 2000 Feb; 117: 613-4.

7. Weymand B, Noel H, Goncette L, Noirhomme P, Collard P. Solitary fibrous tumor of the pleura: a report of five cases diagnosed by transthoracic cutting needle biopsy. Chest 1997; 112: 1424-8.

8. Congregado M, Loscertales J, Jimenez Merchan R, Arroyo Tristan A, Giron Arjona JC, Arenas Linares C. Giant solitary fibrous tumor of the pleura treated by videoassisted surgery: a Case Report. Arch Bronconeumol 2004; 40: 183-4.

9. Tastepe I, Alper A, Ozaydin HA, Memis L, Cetin G. A case of multiple synchronous localised fibrous tumour of the pleura. Eur J Cardiothorac Surg 2000;18: 491-4.

10. Odom SR, Genua JC, Podesta A, Rubin HP. Recurrence of a solitary fibrous tumour of the pleura: a case report. Conn Med 2004; 68: 367-70.

11. Bini A, Grazia M, Stella F, Petrella F, Bazzocchi R. Giant malignant fibrous histiocytoma of he pleura arising from solitary fibrous tumour. Thorax 2004; 59 (6): 544.

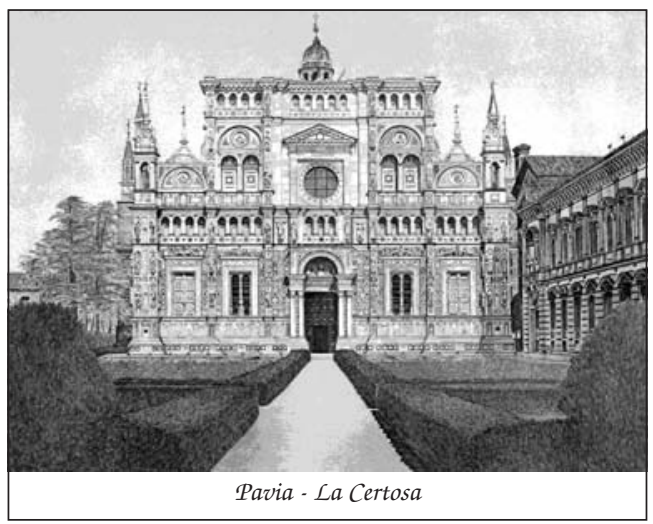

